\title{
How can we keep our psychiatrists?
}

Merryll Vorster graduated and specialised in psychiatry at the University of the Witwatersrand. She is currently Chief Specialist and Associate Professor, Community Psychiatry (joint appointment with the Gauteng Health Department) and Head of the Department of Neurosciences at Wits, and has previously worked at Sterkfontein,

J G Strydom, Tara and South Rand hospitals. She is President-Elect of the South African Society of Psychiatrists.

An article in the Citizen of 5 November 2002 stated that 3351 doctors, dentists and pharmacists resigned from the employ of Gauteng Health during 2000 and 2001 . Where did they all go?

There are 355 psychiatrists practising in South Africa. This figure remains constant despite our efforts at training some 20 new psychiatrists per year.

It is likely that newly qualified specialists stay within state employment, usually in academic joint posts, for a year or two until moving (some of them) into private practice. Many emigrate from private practice, and less often directly from state. Only a small percentage of these return.

Why do state-employed psychiatrists resign? It is my opinion that financial considerations play only a small role, and that dissatisfaction with working conditions, while cited, is also not pivotal in making such a decision. The sense of being undervalued and merely a cog in an inefficient bureaucratic organisation is the major problem.

Doctors will put up with long working hours in poor conditions if they feel needed and valued. They are generally caring people - that is why they chose the medical profession in the first place. But when they are confronted with administrative hurdles, and made to feel as if they are both inessential and a burden to the system, thoughts of leaving are born.

In South Africa the culture of respect for the old, the wise, the teachers and the healers is missing, and we need to ask ourselves how this came about. Other countries are actively recruiting our intellectuals and skilled professionals, so clearly there is nothing

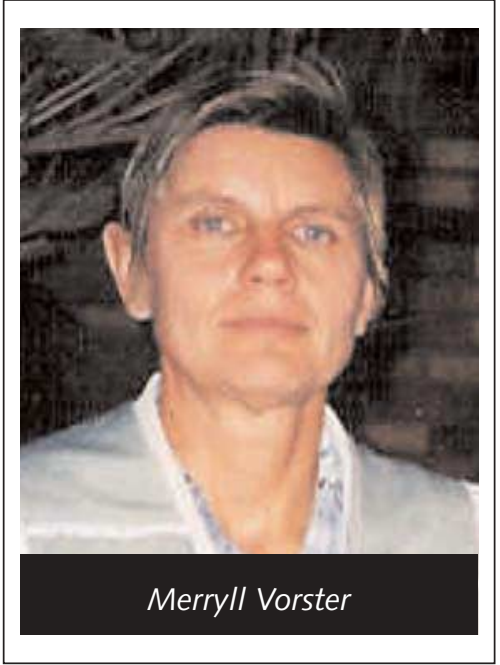

wrong with our training processes.

Why do psychiatrists in private practice leave? Although they may have better facilities than their colleagues in state employment, they work long hours due to patient demand. There are few specialists to choose from, so appointment diaries are always full. They too have administrative woes; they have great difficulty extracting their fees from medical aids, and often have to motivate for medication they prescribe, or for clinic admissions when required.

Job offers are constantly being made which seem attractive, especially in the context of the high crime rate in South Africa and the ever-increasing prices of goods and petrol.

So, why stay?

South Africa offers a lifestyle unsurpassed by other countries. 
There are unlimited work opportunities, and for the capitalists, the chance to make a lot of money. Psychiatrists in South Africa will see pathology and have hands-on experience of conditions that professionals in other countries can only read about in dry texts. I have had the personal experience of being disbelieved when I gave my statistics for interviewing serial killers per annum - the numbers were comparable to lifetime figures for others. There is a diversity of psychiatric fields, and unlimited opportunities to study within them. There is a needy patient population. There is the chance to make a difference by thinking creatively to solve problems that don't get less when colleagues leave, but only get worse.

And of course there are the constant opportunities to test our own abilities to cope with stress!

How do we address the problem?

Although each professional has the right to live and work where he or she chooses, active recruitment should be discouraged. Senior staff in all sectors should make the time and effort to mentor junior staff, so that at least they can be nurtured. Administrative issues should be sorted out as far as possible. Academic interests should be maintained. CPD should be viewed not as the enemy, or merely as a one-point-per-hour feeding scheme, but as a forum to allow individuals to ventilate their feelings and discuss solutions.

How do you think we can keep senior staff?

Please fill your solution(s) in the box and post it to $\mathrm{PO}$ Box 73658 , Fairland, 2030. Any contributions will be appreciated.

\section{Merryll Vorster}

President-Elect, South African Society of Psychiatrists

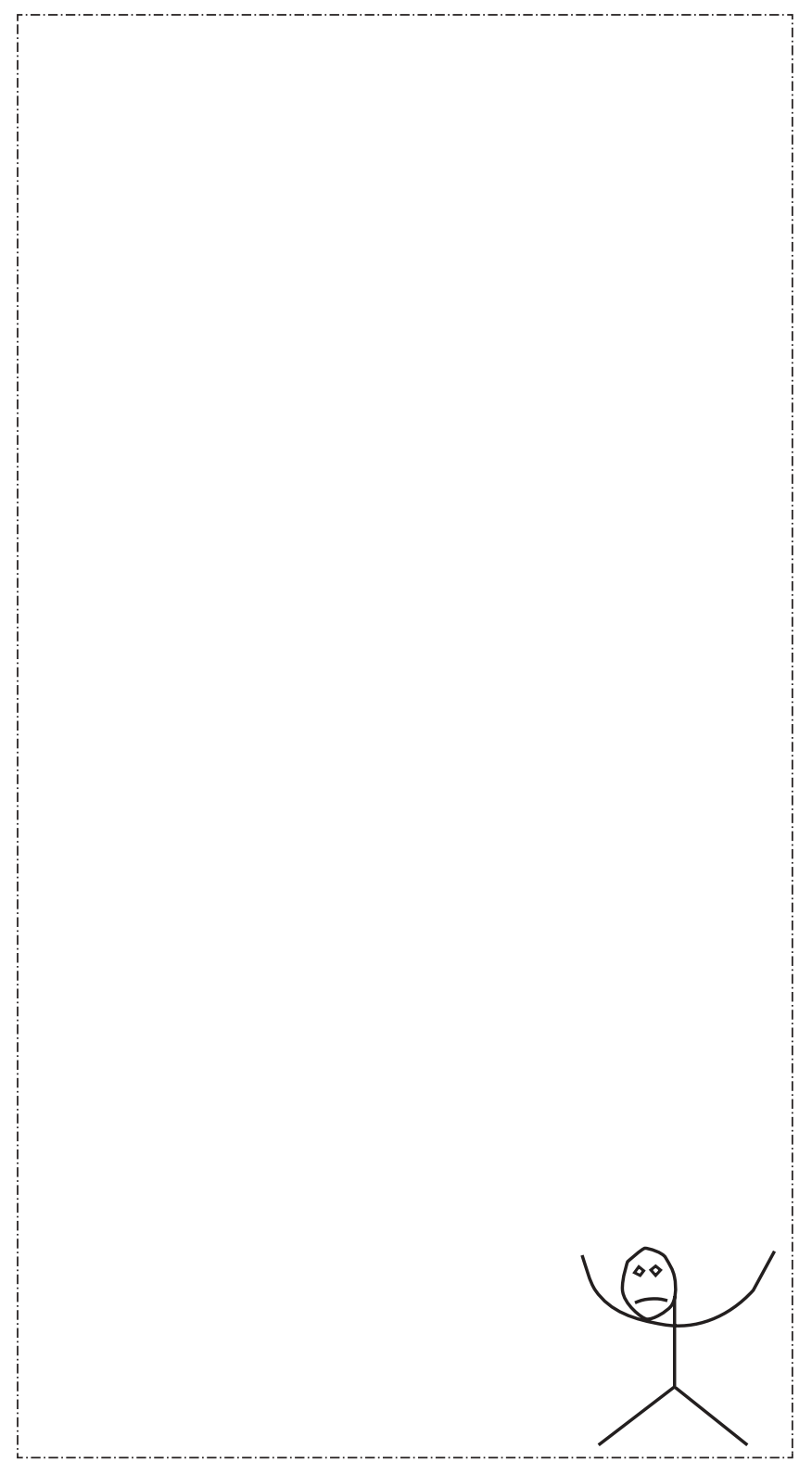

